

\title{
STATISTICAL FILTERING OF SPACE NAVIGATION MEASUREMENTS
}

August 1963

\begin{abstract}
Note: This report has aleo been isaued as AIA Preprint No. 63-333, presented at the AlAA Guidance and Control Conference at Massachusetts Institute of Technology August 12-14, 1963.
\end{abstract}

\section{EXPERIMENTAL ASTRONOMY LABORATORY MASSACHUSETTS INSTITUTE OF TECHNOLOGY CAMBRIDGE 39, MASSACHUSETTS \\ Prepared for Publication by Jackson \& Moreland, Inc.}


TABLE OF CONTENTS

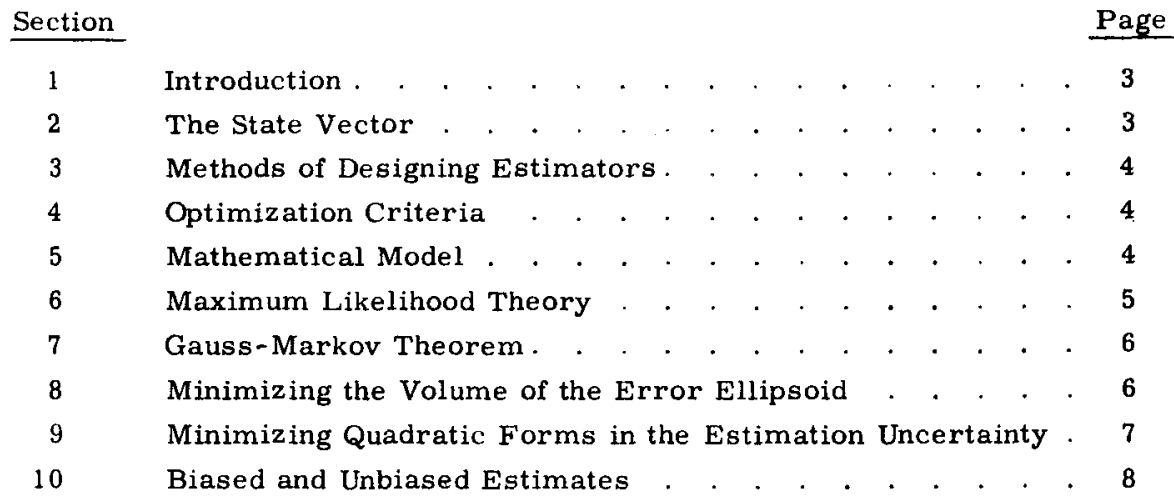

\section{APPENDIX}

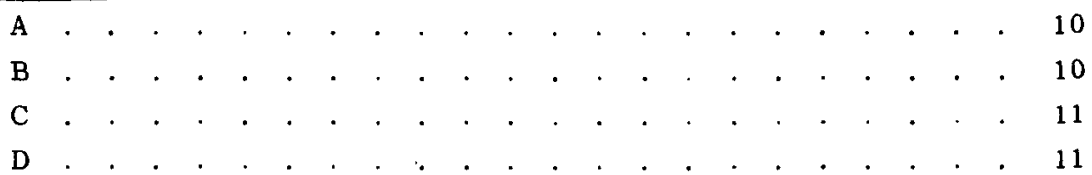




\section{STATISTICAL FILTERING OF SPACE NAVIGATION MEASUREMENTS ${ }^{1}$}

James E. Potter ${ }^{2}$

Robert G. Stern ${ }^{3}$

Massachusetts Institute of Technology

\section{Abstract}

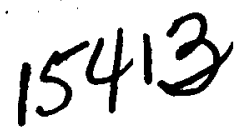

The criteria that have been used for designing an estimator which computes the state vector of a space vehicle from redundant navigational measurements are reviewed and compared. It is shown that under the assumption of linearity all these criteria lead to the same estimator. (or filter). . If all measurement uncertainties have Gaussian distributions, it is shown that the estimate obtained from optimum filter theory is identical with the maximum likelihood estimate.

A proof is presented that the Bayes estimator, which is biased in favor of the initial conditions, leads to the same result that would be obtained from an unbiased estimator to which six pseudo-measurements, representing the inftial conditions, have been added.

Although the theory has been applied in the epecific area of space navigation, the results obtained are generally applicable to the estimation of multi-dimensional random variables.

\section{Nomenclature}

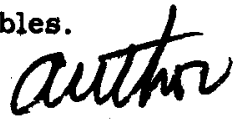

\section{General Notation}

Underlining a lower-case letter indicates that the letter represents a column vector.

An asterisk over a capital letter indicates that the letter represents a matrix:

Enclosing a matrix within two vertical bars indicates the determinant of the matrix.

Angular brackets < $>$ indicate the average value of the bracketed quantity.

\footnotetext{
${ }^{1}$ The analysis in this paper represents one phase of research carried out for the National Aeronautics and Space Administration under Contracts NAS 9-153 and NoG 254-62.

${ }^{2}$ Staff Engineer, Ingtrumentation Laboratory

3 Staff Engineer, Experimental Astronomy Laboratory
}

Superscript $\mathrm{T}$ following a vector or matrix indicates the transpose of the vector or matrix. Superscript -1 following a square matrix indicates the inverse of the matrix.

\section{Symbols}

A symmetric matrix (in Section 7 and Appendix C)

A $\quad r$-by-a matrix (In Section 9)

A positive semidefinite $r$-by-r matrix (in Appendices $B$ and $D$ )

A $_{1} \quad r$-by-r auxiliary matrix (in Appendix D)

b mean value of positive semidefinite quadratic form which is to be minimized

B gymmetric matrix (in Section 7 and Appendix C)

* B-by-r matrix (in Section 9)

B $\quad r$-by-s matrix (in Appendix B)

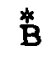
positive semidefinite $r-b y-r$ matrix (in Appendix D)

$\stackrel{\text { C }}{\mathrm{ji}}$

$d_{i}$

$\mathrm{n}$-by-n state transition matrix

$i$-th diagonal element of $D$

D diagonal matrix

$\mathrm{D}_{1}$ auxiliary diagonal matrix

e error vector associated with the estimated state vector $\hat{\underline{x}}$

$\underline{\mathrm{e}} \mathrm{B}$

error vector associated with $\hat{\underline{x}}_{B}$

* covariance matrix associated with $\underline{e}$

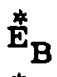

${ }_{\mathbf{U}}^{*}$ covariance matrix associated with $\underline{e}_{B}$ covariance matrix assoclated with error in $\hat{\underline{x}}_{\mathbf{U}}$

${ }_{\text {F }}$ arbitrary unbiased filter F $_{B}$ arbitrary biased filter

${ }_{\mathbf{B}}^{*}$ unbiased filter which has same covariance matrix as $\mathrm{F}_{\mathrm{B}}$ 


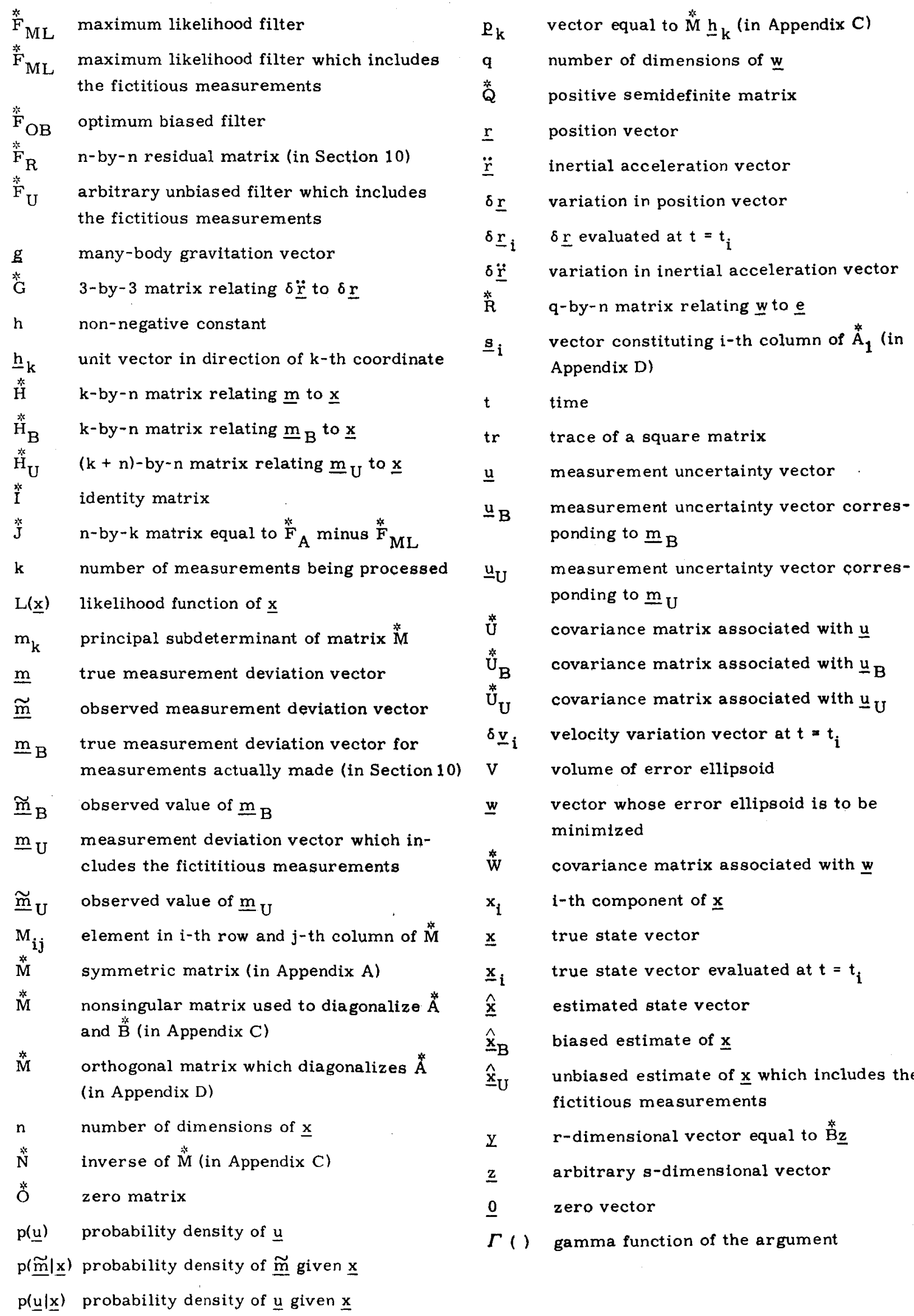




\section{Subscripts}

$\begin{array}{ll}\text { A } & \text { relating to filter }{ }_{A}^{*} \\ \text { ML } & \text { relating to maximum likelihood filter }{ }_{M L}^{*} \\ \text { OB } & \text { relating to optimum biased filter }{ }_{\mathrm{F}}^{*}\end{array}$

1. Introduction

The vector equation of motion of a space vehicle in free night in a many-body gravitational field is

$$
\ddot{\underline{r}}=\mathbf{g}(\underline{r}, t)
$$

The inertial acceleration $\underline{\underline{\underline{ }}}$ is due entirely to the many-body grevitation vector $g$, which is a function of both spatial position and time.

In the usual guidance analysis Equation (1-1)

is linearized by assuming that the vehicle's actual position and velocity at any specified time differ only slightly from the pre-computed nominal, or reference, position and velocity at that time. If the symbol $\delta$ is used to signify the variation of a quantity from its nominal value, the linearized equations of motion of the vehicle can be written in matrix form as follows:

$$
\delta \underline{\ddot{r}}=\stackrel{k}{G} \delta \underline{r}
$$

Here $\delta \underline{\ddot{r}}$ and $\delta \underline{\mathbf{r}}$ are three-component column vectors and $G$ is a 3-by-3 matrix which is shown in Reference (1) to be symmetric for any gravitational field. The elements of $G$ are functions of the characteristics of the reference trajectory and are independent of the components of $\delta \underline{r}$.

Equation (1-2) represents three secondorder linear differential equations with variable coefficients. The solution of the system contains six constants of integration. When the mathematical model is specifled and the reference trajectory has been pre-computed, the motion of the vehicle along its actual trajectory can be completely determined once the six integration constants have been evaluated. The object of this paper is to examine techniques for estimating the integration constants from a set of redundant measurements, each of which is corrupted by noise.

\section{The State Vector}

The integration constants are conveniently grouped in the six-component column vector $x_{i}$, known as the "state vector," which consists of the three components of $\delta \underline{r}_{i}$ and the three components of the velocity variation vector $\delta \underline{v}_{\mathfrak{i}}$ at some specified time $t_{i}$.

$$
\underline{x}_{i}=\left[\begin{array}{l}
\delta \underline{r}_{i} \\
\delta \\
\underline{v}_{i}
\end{array}\right]
$$

Various values of $t_{i}$ have been used in the literature to define the particular state vector in terms of which the actual motion of the vehicle is expressed. For example, in Reference $(2) t_{i}$ is the time of cut-off of the main propulsion system and thus the time of initiation of the free-fall trajectory; in References (3) and (4) $t_{i}$ is the "current" time, that is, the time at which the most recent measurement was made or the time at which the next measurement is to be made; and in Reference (5) $t_{i}$ is the nominal time of arrival at the destination.

The goal of the data processing is to minimize the errors in the estimate of the components of $x_{i}$. It is shown in the later sections of this paper that, for the estimation techniques considered, the filter which minimizes the r.m.s. uncertainties in the components of the state vector at one specified time minimizes the r.m.s, uncertainties in the components of the state vector at any other time.

If position variation is more significant than velocity variation, as is the case for the destination state vector when the mission objective is to pass through a fixed point in inertial space at a fixed time, one might hope to get a more accurate estimate of position by designing a filter which minimizes the r.m.s. position uncertainty without regard for velocity uncertainty. It will be shown that the estimator which minimizes the r.m.s. uncertainty in the state vector also minimizes the r.m.s. uncertainty of any sub-vector of the state vector, and thus no improvement in the estimate can be achieved by neglecting the variation in velocity.

This concept can be extended to show that the same estimate is obtained even if the six integration constants being considered consist of the components of position variation at one time (e.g., the time of arrival at the destination) and the components of velocity variation at a different time (e. g., the "current" time). 


\section{Methods of Designing Estimators}

The conventional methods of designing estimators for a multi-dimensional random variable fall into two main categories - the method of maximum likelihood and the technique based on optimum filter theory.

The maximum likelihood method was developed by statisticians and is based on the concept of maximizing a particular conditional probability. Practical estimators can be obtained from the method only by assuming that the individual measurements are normally distributed.

The object of optimum filter theory is to

find a linear estimator which minimizes some function of the variances and covariances of the uncertainties in the estimated state vector. The development of the theory does not depend on the assumption that the uncertainties in the measurements are normally distributed.

Irrespective of whether maximum likelihood theory or optimum filter theory is used, it is possible to obtain either an "unbiased" or a "biased" estimate. An unbiased estimate is one in which only the direct measurements are used and no assumption is made about the a priori distribution of the uncertainties in the components of the state vector. The unbiased estimator is characterized by the fact that the estimated state vector coincides with the true state vector if all measurements are perfect. A biased, or Bayes, estimate is one in which the a priori distribution of the uncertainties in the components of the state vector is taken into account.

\section{Optimization Criteria}

There are three general types of criteria that have been used in applying optimization techniques to the redundant data problem. The first type, which is relevant to the method of maximum likelihood, consists of determining that state vector $\mathrm{x}$ which maximizes the probability density of the occurrence of the measurements that have actually been obiained. This probability density is known as the likelihood function.

The second type of criterion involves minimizing the volume of the error ellipsoid associated with some linear function of the uncertainties in the components of the estimated state vector. If $\mathrm{e}$ is the $\mathrm{n}$-dimensional column vector containing these uncertainties and if the vector $\underline{w}$ is given by

$$
\underline{w}={ }^{*} \mathrm{R} \underline{\mathbf{e}}
$$

then the error ellipsoid in $\underline{w}$-space is defined by the equation

$$
\underline{w}^{T} \stackrel{*}{W}^{-1} \underline{w}=1
$$

where $\stackrel{*}{W}$ is the covariance matrix associated with w.

$$
\stackrel{\vec{w}}{\mathrm{w}}=\left\langle\begin{array}{ll}
\underline{w} & \underline{w}^{\mathrm{T}}
\end{array}\right\rangle
$$

For example, if $\underline{w}$ is equal to $\underline{e}, \stackrel{R}{R}$ becomes the identity matrix; if $\underline{w}$ consists of only that portion of e containing the uncertainties in position, $\mathrm{R}$ is the following 3-by-6 matrix.

$$
\stackrel{\mathrm{r}}{\mathrm{R}}=\left[\begin{array}{llllll}
1 & 0 & 0 & 0 & 0 & 0 \\
0 & 1 & 0 & 0 & 0 & 0 \\
0 & 0 & 1 & 0 & 0 & 0
\end{array}\right]
$$

The third type of criterion minimizes the mean value of a positive semidefinite quadratic form in the uncertainties of the estimated state vector. In mathematical notation, the quantity to be minimized is

$$
\mathrm{b}=\left\langle\underline{\mathrm{e}}^{\mathrm{T}} \underline{\mathrm{Q}} \underline{\mathrm{e}}\right\rangle
$$

where $Q^{*}$ is a positive semidefinite matrix.

\section{Mathematical Model}

The number of measurements being processed is designated $\mathrm{k}$; these measurements are combined in a $\mathrm{k}$-dimensional column vector $\underline{\mathrm{m}}$. In order to justify the use of linear theory, the components of $m$ are the deviations of the measured values from those values which would be observed ideally if the vehicle were on its reference trajectory.

In the general case $\underline{x}$, the parameter vector to be estimated, is of dimension $n$. Since it is assumed that redundant data are available, $k$ is greater than $n$. For the state vector in space navigation $\mathrm{n}$ is equal to six.

The vector $\underline{m}$ is assumed to be linearly related to $x$ in a manner that is a known function of the reference trajectory and of the type and time of each measurement. 


$$
\mathrm{m}=\stackrel{*}{\mathrm{H}} \underline{\mathrm{x}}
$$

* is a k-by-n matrix whose elements are precomputed. The rank of $\mathrm{H}$ must be equal to $n$, for, if this were not $80, x$ could not be determined from $\mathrm{m}$.

The observed value of the measurement vector is $\underline{\underline{m}}$, which differs from the true value due to the uncertainties in the individual measurements. The difference between $\underline{\underline{m}}$ and $\underline{m}$ is equal to the measurement uncertainty vector $\underline{u}$.

$$
\underline{\underline{m}}=\underline{m}+\underline{u}=\stackrel{k}{H} \underline{x}+\underline{u}
$$

The covariance matrix of the measurement uncertainties is the symmetric k-by-k matrix Ü.

$$
\text { * }=\left\langle\begin{array}{ll}
\underline{\mathrm{u}} & \underline{\mathrm{u}}^{\mathrm{T}}
\end{array}\right\rangle
$$

For any practical case $\mathrm{U}$ is a positive definite matrix.

The estimate of the state vector obtained from any of the filters to be developed is designated $\hat{\underline{x}}$. The error in the estimate is $\underline{e}$.

$$
\underline{e}=\underline{\hat{x}}-\underline{x}
$$

The covariance matrix of the components of $e$ is $\mathrm{E}$.

$$
\text { E }=\left\langle\underline{e} \underline{e}^{T}\right\rangle
$$

\section{Maximum Likellhood Theory}

The likelihood function $L(\underline{x})$, in mathematical terms, is

$$
L(\underline{x})=p(\underline{\tilde{m}} \mid \underline{x})
$$

where $p(\tilde{\underline{m}} \mid \underline{x})$ is the probability density of the observed measurement vector $\tilde{\underline{m}}$, given the state vector $\underline{x}$. From Equation (5-2), $\underline{\tilde{m}}$ is the sum of a determiniatic function of $\underline{x}$ and the random vector u. Therefore,

$$
p(\underline{\tilde{m}} \mid \underline{x})=p(\underline{u} \mid \underline{x})
$$

The probability density of $\underline{u}$ is independent of $\underline{x}$, so that

$$
L(\underline{x})=p(\underline{u})
$$

$p(\underline{u})$ is the joint probability density of the components of $\underline{u}$. The components of $\underline{u}$ are assumed to have a joint Gaussian distribution. Then,

$$
L(\underline{x})=\frac{\exp \left(-\frac{1}{2} \underline{u}^{T}{ }_{U}^{-1} \underline{u}\right)}{\left[(2 \pi)^{k}|U|\right] 1 / 2}
$$

where $|\stackrel{*}{U}|$ is the determinant of $\mathrm{U}$.

Since $\log \mathrm{L}(\underline{\mathrm{x}})$ is a monotonically increasing function of $L(\underline{x})$, maximizing the logarithm with respect to $\underline{x}$ yields the same value of $x$ as maximizing the likelihood function itself.

$$
\begin{aligned}
& \log L(\underline{x})=-\frac{1}{2} \log \left[(2 \pi)^{k}\left|{ }^{*} U\right|\right]-\frac{1}{2} \underline{u}^{T}{ }^{*}{ }^{-1} \underline{u}
\end{aligned}
$$

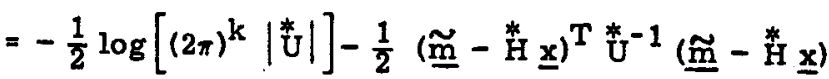

The partial derivative of $\log \mathrm{L}(\underline{\mathrm{x}})$ with respect to $x_{i}$, one of the components of $\underline{x}$, when all other components of $\underline{x}$ are held constant, is

$$
\begin{aligned}
\frac{\theta \log L(\underline{x})}{\partial x_{i}}= & \frac{1}{2}\left[\frac{\partial \underline{x^{T}}}{\partial x_{i}}{ }_{H}^{*} T^{* 1}(\underline{m}-\stackrel{*}{H} \underline{x})\right. \\
& \left.+(\underline{m}-\stackrel{*}{H} \underline{x})^{T} U^{-1} \stackrel{*}{H} \frac{\partial \underline{x}}{\partial x_{i}}\right]
\end{aligned}
$$

The second term on the right-hand side of Equation (6-6) is the transpose of the first term. Since both terms are scalar quantities, they are equal to each other.

$$
\begin{aligned}
& \frac{\partial \log L \underline{\underline{x}})}{\partial x_{i}}=\frac{\partial \underline{x^{T}}}{\partial x_{i}} H^{*} \stackrel{U}{H}^{-1}(\underline{\tilde{m}}-\stackrel{*}{H} \underline{x}) \quad(6-7) \\
& \text { The partial derivative } \frac{\partial \underline{x^{T}}}{\partial x_{i}} \text { is a row vec- }
\end{aligned}
$$

tor with a one in the $i$-th position and all other components zero. Therefore, the right-hand side of Equation (6-7) represents the $i$-th component of the column vector $H^{*}{ }_{U}^{* 1}(\underline{\tilde{m}}-\stackrel{*}{H} \underline{x})$. When the likelihood function is a maximum, each of the $n$ partial derivatives of $\log L(\underline{x})$ with respect to the components of $\underline{x}$ vanishes. Then,

$$
{ }_{\mathrm{H}}^{*} \mathrm{~T} \mathrm{U}^{-1}(\underline{\tilde{m}}-\stackrel{*}{\mathrm{H}} \underline{\underline{x}})=\underline{0}
$$

The solution of $(6-8)$ for $\underline{x}$ is $\underline{\hat{x}}_{M L}$, the maximum likelihood estimate of $\underline{x}$.

$$
\underline{\hat{x}}_{M L}=\stackrel{F L}{\tilde{m}}^{*}
$$

where

$$
{ }_{\mathrm{ML}}^{*}=\left({ }_{\mathrm{H}}^{*}{ }_{\mathrm{U}}^{-1} \stackrel{*}{\mathrm{H}}\right)^{-1}{ }_{\mathrm{H}}^{*} \mathrm{~T}_{\mathrm{U}}^{* 1}
$$


It may be noted tha.

$$
\stackrel{\ddot{F}}{\text { FIL }}_{\text {H }} \stackrel{\ddot{H}}{\vec{I}}
$$

so that the maximum likelihood filter $\stackrel{\stackrel{*}{F}}{F}_{M L}$ is an unbiased filter.

From Equations (5-2) and (6-9),

$$
\begin{aligned}
& \hat{\underline{x}}_{M I L}=\stackrel{*}{F}_{M L} \stackrel{*}{H} \underline{x}+\stackrel{*}{*}_{M L} \underline{u} \\
& =\underline{x}+\stackrel{\psi k}{F}_{M I L} \underline{u}
\end{aligned}
$$

The error $\underline{e}$ IL in the maximum likelihood estimate is

$$
\underline{e}_{M L}=\hat{\hat{x}}_{M L}-\underline{x}=\stackrel{*}{F}_{M L} \underline{u}
$$

The covariance matrix $\stackrel{\text { F }}{E}_{M L}$ is then given by

$$
\begin{aligned}
& \stackrel{*}{E}_{M L}=\left\langle\underline{e}_{M L} \stackrel{e}{-}_{M L}^{T}\right\rangle \\
& =\stackrel{*}{F}_{M L} \stackrel{*}{U} \stackrel{*}{F}_{M L}^{T} \\
& =\left(\mathrm{H}^{*}{ }^{*}-1{ }_{\mathrm{H}}^{*}\right)^{-1}
\end{aligned}
$$

\section{Gauss-Markov Theorem}

If $\stackrel{*}{\mathrm{~A}}$ and $\stackrel{\mathrm{B}}{\mathrm{B}}$ are symmetric matrices, $\mathrm{A}^{*}$ will be said to be greater than $B$ if the matrix difference $(A-B)$ is positive definite. Similarly $A \geq B$ will indicate that $(\stackrel{A}{A}-\vec{B})$ is positive semidefinite. The standard definitions of "positive definite" and "positive semidefinite" are given in Appendix A.

It is important to note that $A$ 像 does not imply that each element of $A$ is greater than the corresponding element of $\mathrm{B}$. For example,

$$
\left[\begin{array}{ll}
4 & 0 \\
0 & 4
\end{array}\right]>\left[\begin{array}{ll}
2 & 1 \\
1 & 2
\end{array}\right]
$$

even though the off-diagonal elements of the righthand matrix are larger than the off-diagonal elements of the left-hand matrix.

The Gauss-Markov theorem states that under the assumptions postulated in Section 5 些 is less than or equal to the $\mathrm{E}$ matrix for any other unbiased estimator. This theorem indicates that the variance of the component of the estimation uncertainty in any given direction in state space is minimized by the maximum likelihood estimate. To prove the theorem, let $\vec{F}_{A}^{*}$ be an arbitrary unbiased filter. The corresponding state vector $\underline{\hat{x}}_{\mathrm{A}}$ is

$$
\underline{\hat{x}}_{A}=\stackrel{* k}{F}_{A}(\stackrel{* k}{H} \underline{x}+\underline{u})
$$

Since the filter is unbiased,

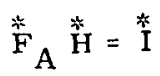

Then, the estimation error $\underline{e}_{\mathrm{A}}$ is

$$
\underline{\mathrm{e}}_{\mathrm{A}}=\underline{\hat{x}}_{\mathrm{A}}-\underline{x}_{\mathrm{A}}=\stackrel{*}{\mathrm{~F}}_{\mathrm{A}}
$$

The covariance matrix $\stackrel{*}{\mathrm{E}}_{A}$ is given by

$$
\stackrel{*}{\mathrm{E}}_{\mathrm{A}}=\left\langle\underline{e}_{\mathrm{A}} \underline{e}_{\mathrm{A}}^{\mathrm{T}}\right\rangle=\stackrel{*}{\mathrm{~F}}_{\mathrm{A}} \stackrel{*}{\mathrm{U}} \stackrel{*}{\mathrm{~F}}_{\mathrm{A}}^{\mathrm{T}}
$$

If $\mathrm{J}$ is defined by

$$
\mathrm{J}^{*}=\mathrm{F}_{\mathrm{A}}-\stackrel{*}{\mathrm{~F}}_{\mathrm{ML}}
$$

the product $\stackrel{\mathrm{J}}{\mathrm{H}}$ is

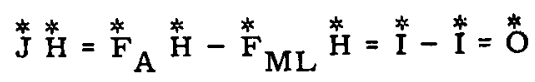

Substituting $\stackrel{*}{F}_{A}=\stackrel{*}{J}+\stackrel{*}{F}_{M L}$ into $(7-4)$ yields

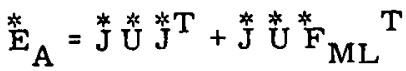

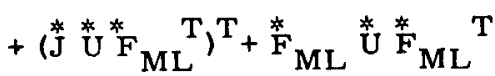

From Equations (6-10) and (7-6),

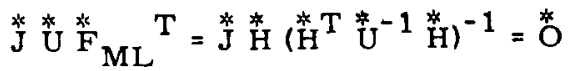

From Equations (6-14), (7-7), and (7-8),

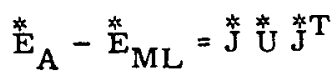

In Appendix $B$ it is shown that, since $U$ is positive definite, $\mathrm{J} U \mathrm{U}$ Therefore,

$$
\mathrm{E}_{\mathrm{A}} \geq \text { 窄 }_{\mathrm{ML}}
$$

Because $\mathrm{U}$ is positive definite, $\mathrm{J}_{\mathrm{U}}^{*} \mathrm{~J}$ can be zero only if $\mathrm{J}^{+}$is the zero matrix. Consequently, ${ }_{\mathrm{E}}$ can be equal to $\mathrm{E}_{\mathrm{ML}}$ only if $\mathrm{F}_{\mathrm{A}}$ is equal to $\mathrm{F}_{\mathrm{ML}}$.

It may be noted that in this derivation it has not been necessary to assume that the measurement uncertainties have a joint Gaussian distribution.

\section{Minimizing the Volume of the Error Ellipsoid}

The first type of optimum filter to be developed is that which minimizes the volume of the 
error ellipsoid in $\underline{w}$-space. The equation of the error ellipsoid is (4-2). Its volume is

$$
\mathrm{V}=\frac{\pi^{\frac{\mathrm{g}}{2}}|\stackrel{*}{\mathrm{~W}}| 1 / 2}{\Gamma\left(\frac{\mathrm{q}}{2}+1\right)}
$$

where $\underline{w}$ is a $q$-dimensional vector, It is apparent that, for a given value of $q$, the volume of the ellipsoid is a function of the square root of the determinant of the covariance matrix W. Thus, minimizing the ellipsoidal volume is equivalent to minimizing $|\stackrel{*}{w}|$.

By the use of Equations $(4-1)$ and $(4-3)$ W may be written in terms of $\mathrm{R}$ and $\mathrm{E}$.

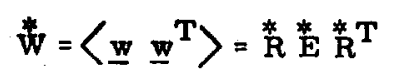

If $\mathrm{E}_{\mathrm{ML}}$ and $\mathrm{E}_{\mathrm{A}}$ are, respectively, the covariance matrices associated with the uncertainties in the maximum likelihood estimator and with any other unbiased estimator, then from the Gauss-Markov theorem ( $\left.{ }_{A}-\underline{E}_{M L}\right)$ is positive semidefinite. In Appendix B. It is shown that, if ( ${ }_{A}^{*}-E_{M L}$ ) is positive semidefinite, the matrix product $\frac{M}{R}\left(E_{A}-E_{M L}\right) h^{T}$, which is equal to ( $\mathrm{W}_{A}-\mathrm{W}_{M L}$ ), is also positive semidefinite. Finally, from Appendix C.

$$
\left|W_{\mathrm{A}}\right| \geq\left|\mathrm{W}_{\mathrm{ML}}\right|
$$

Therefore, use of the maximum likelihood estimator results in the minimum volume of the error ellipsoid in $\mathbf{w}$-space.

It may be shown that if $\stackrel{*}{W}_{A} \geq \stackrel{*}{W}_{M L}$ the error ellipsoid corresponding to the maximum likelihood estimator is contained in the error ellipsoid corresponding to the other estimator.

I Wo that

If the eatimator ${ }_{\mathrm{F}_{\mathrm{A}}}$ actually minimizes

$$
\left|\stackrel{*}{\mathbf{W}}_{\mathbf{A}}\right|=\left|\stackrel{*}{\mathbf{W}}_{\mathrm{ML}}\right|
$$

then from Appendix $C$ the two $\stackrel{*}{W}$ matrices are equal.

$$
\stackrel{\star}{\mathrm{W}}_{\mathbf{A}}=\stackrel{\star W}{\mathrm{~W}}_{\mathrm{ML}}
$$

Thus,

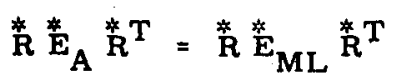

If $q=n$, so that $\vec{R}$ is of rank $n$ and can be inverted,

$$
\stackrel{-*}{\mathrm{E}}_{\mathrm{A}}={ }_{\mathrm{ML}}
$$

In Section 7 it was shown that Equation (8-7) implies that

$$
\text { F }_{\mathbf{A}}=\stackrel{*}{\mathbf{F}}_{\mathrm{ML}}
$$

Therefore, when $q=n$, the optimum filter is unique.

If $q<n$, there may be a family of filters $\mathrm{F}_{A}$, only one of which is equal to $\mathrm{F}_{\mathrm{ML}}$, all satisfying the criterion that $W^{*}$ be minimized.

One possible type of $\mathrm{R}$ matrix for the case $q=n$ is the state transition matrix $C_{j i}$ which transforms the state vector $\underline{x}_{i}$ at time $t_{i}$ to the state vector $\underline{x}_{j}$ at time $t_{j}$.

$$
\underline{x}_{j}=\stackrel{*}{C}_{j i} \underline{x}_{i}
$$

Then $\underline{e}$ is the error vector associated with $\underline{x}_{i}$, and w correspondingly is the error vector associated with $x_{j^{*}}$ Since $q=n$, the unique filter which minimizes the volume of the six-dimensional error ellipsoid associated with the state vector $\underline{x}_{1}$ is also the unique filter which minimizes the volume of the six-dimensional error ellipsoid associated with any other state vector $\underline{x}_{j}$. Thus the time to which the state vector is referred does not affect the accuracy of estimation.

If the $\mathrm{R}$ matrix is a hybrid state transition matrix which transforms $\underline{x}_{i}$ into a vector consisting of the position variation at one time and the velocity variation at another time or into position variations at two different times or into velocity variations at two different times, the same estimate of the state vector $x_{s}$ at some arbitrary time $t_{s}$ is obtained as long as $R$ is nonsingular.

If the error ellipsoid to be minimized is associated with only the position components of some state vector, $q<n$, and in that case the maximum likelihood filter is only one a set of possible optimum filters.

\section{Minimizing Quadratic Forms in the Estimation Uncertainty}

In Section 4 it was pointed that one of the criteria used in applying optimization techniques is minimizing the statistic

$$
b=\left\langle\underline{e}^{T}{ }^{\mathrm{*}} \mathrm{e}\right\rangle
$$


Several versions of the positive semidefinite matrix $Q$ are of practical importance. If $Q$ is the identity matrix, the square root of $b$ is the r.m.s. value of the magnitude of the error vector $e_{i}$ associated with the state vector $x_{i}$. If $\stackrel{*}{Q}$ is equal to $\stackrel{*}{C}_{j i} T \stackrel{*}{C}_{j i}$, the square root of $b$ is the r.m.s. value of the magnitude of the error vector $\underline{e}_{j}$ associated with $x_{j}$. If $\stackrel{*}{Q}$ is given by

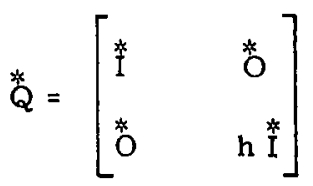

where $h$ is a non-negative constant, the minimization involves velocity variation components that are weighted differently from the position variation components. In particular, if $h=0$, the velocity components are ignored. It is also possible to choose $Q$ such that $b$ is equal to the mean of the sum of the squares of the position estimation uncertainty at one time and the velocity estimation uncertainty at another time.

Since $\mathrm{e}^{\mathrm{T}} \mathrm{Q}$ e is a scalar (a $1-b y-1$ matrix), it is equal to its trace. Then $(9-1)$ becomes

$$
b=\left\langle\operatorname{tr}\left(\underline{e}^{T} \underline{Q} \underline{e}\right)\right\rangle
$$

In Reference 7 it is shown that if $A$ is an $r-b y-s$ matrix and $B$ is an $s-b y-r$ matrix,

$$
\operatorname{tr}(\stackrel{\mathrm{B}}{\mathrm{A}})=\operatorname{tr}(\stackrel{*}{\mathrm{~A}} \mathrm{~B})
$$

Then,

$$
\begin{aligned}
& \mathrm{b}=\left\langle\operatorname{tr}\left[\left(\underline{\mathrm{e}}^{\mathrm{T}} \mathrm{U}^{\mathrm{Q}}\right) \underline{\mathrm{e}}\right]\right\rangle=\left\langle\operatorname{tr}\left(\underline{\mathrm{e}} \underline{\mathrm{e}}^{\mathrm{T}} \underline{\mathrm{Q}}\right)\right\rangle \\
& =\operatorname{tr}\left[\left\langle\underline{e} \underline{e}^{T}\right\rangle \hat{Q}\right]=\operatorname{tr}\left(\frac{*}{E} \hat{Q}\right)
\end{aligned}
$$

It will now be shown that the maximum likelihood filter $\stackrel{\text { F }}{\mathrm{F}}_{\mathrm{ML}}$ minimizes the statistic $b$.

The Gauss-Markov theorem shows that $\left(\mathrm{E}_{\mathrm{A}}-\mathrm{E}_{\mathrm{ML}}\right)$ is positive semidefinite. In Appendix $D$ it is shown that if $\left(\mathrm{E}_{A}-\mathrm{E}_{M L}\right)$ and $\mathrm{Q}$ are both positive semidefinite,

$$
\operatorname{tr}\left[\left(\stackrel{*}{\mathrm{E}}_{\mathrm{A}}-{\stackrel{*}{\mathrm{E}_{\mathrm{ML}}}}_{\mathrm{M}}\right) \stackrel{\mathrm{*}}{\mathrm{k}}\right] \geq 0
$$

Thus,

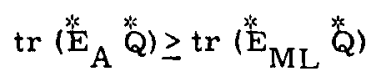

or

$$
\mathrm{b}_{\mathrm{A}} \geq \mathrm{b}_{\mathrm{ML}}
$$

When filter ${ }_{A}$ minimizes $b$, so that $b_{A}$ is equal to $b_{M L}$, then

$$
\operatorname{tr}\left[\left(\stackrel{*}{\mathrm{E}}_{\mathrm{A}}-\stackrel{*}{\mathrm{E}}_{\mathrm{ML}}\right) \stackrel{*}{\mathrm{Q}}\right]=0
$$

If in addition $\stackrel{*}{Q}$ is positive definite, then from Appendix $\mathrm{D}$,

$$
\mathrm{E}_{\mathrm{A}}=\text { 篅 }_{\mathrm{ML}}
$$

From Section 7, Equation (9-10) implies that

$$
\stackrel{*}{\mathrm{~F}}_{\mathrm{A}}=\stackrel{*}{\mathrm{~F}}_{\mathrm{ML}}
$$

This equation indicates that the optimum filter is unique as long as $\frac{\mathrm{C}}{\mathrm{Q}}$ is positive definite.

In Sections 6, 8, and 9, three different optimization criteria have been investigated. It hes been shown that, regardless of which of the three criteria is used, the same optimum filter is obtained. This statement is true when any linearly independent set of six integration constants is used to define the vehicle's variant motion.

\section{Biased and Unbiased Estimates}

In this section it is proved that an unbiased estimator based on $n$ fictitious measurements in addition to the $k$ actual measurements can be constructed with the same covariance matrix $\mathrm{E}$ as a given biased estimator based on the $k$ actual measurements. Conversely, a biased estimator can be constructed which has the same covariance matrix as a given unbiased estimator in which the $n$ fictitious measurements have been taken into account. As a consequence of these facts, there is an optimum biased estimator ${ }_{\mathrm{F}} \mathrm{OB}$ which simultaneously minimizes the volumes of all error ellipsoids and also the means of all positive semidefinite quadratic forms in $\underline{e}$.

Biased estimators are important because they utilize available information in addition to that which is obtained from the direct measurements: The additional information is in the form of the first and second moments of the a priori uncertainty of the state vector $\underline{x}$.

When the optimum biased estimator is used, the resulting estimate of $x$ is more accurate than that which can be obtained from the optimum unbiased estimator ${ }_{\mathrm{ML}}^{\mathrm{*}}{ }_{\mathrm{ML}}$, in the sense that ${ }_{\mathrm{O}}^{\mathrm{k}} \mathrm{OB} \leq$ $\mathrm{E}_{\mathrm{ML}}$ and $\mathrm{b}_{\mathrm{OB}} \leq \mathrm{b}_{\mathrm{ML}}$.

The measurement deviation vector which relates only to the measurements actually made will 
be designated $\underline{m}_{B}$ for biased estimation. The $k$-by- The covariance matrix $\underline{U}_{U}^{*}$ is $n$ matrix relating $\underline{m}_{B}$ to $\underline{x}$ is $\stackrel{k}{H}_{B}$.

$$
\underline{m}_{B}=\stackrel{*}{H}_{B} x
$$

Correspondingly, the measurement uncertainty vector is $\underline{u}_{\mathrm{B}}$ and its covariance matrix is $\mathrm{t}_{\mathrm{B}}$. ${ }_{\mathrm{F}}^{*}{ }_{\mathrm{B}}$ is the blased filter that is to be used.

$$
\begin{aligned}
\overrightarrow{\mathrm{U}}_{\mathrm{U}} & =\left\langle\underline{\underline{u}}_{\mathrm{U}} \underline{\underline{u}}_{\mathrm{U}}^{\mathrm{T}}\right\rangle \\
& =\left[\begin{array}{cc}
\stackrel{\mathrm{U}}{\mathrm{B}}_{\mathrm{B}} & -\left\langle\underline{\underline{u}}_{\mathrm{B}} \underline{\underline{x}}^{\mathrm{T}}\right\rangle \\
-\left\langle\underline{\mathrm{x}}_{\mathrm{B}}^{\mathrm{T}}\right\rangle & \left\langle\underline{\mathrm{x}}^{\mathrm{T}}\right\rangle
\end{array}\right] .
\end{aligned}
$$

$$
\hat{\underline{x}}_{B}=\stackrel{*}{F}_{B} \underline{\tilde{m}}_{B}
$$

where $\underline{\hat{\underline{x}}}_{\mathrm{B}}$ is the blased estimate of the state vector. It ls assumed a priorl that the mean value of $\underline{x}$ ls the zero vector. The error vector for $\underline{\hat{x}}_{B}$ is $\underline{e}_{B}$, which is deftned by

$$
\underline{e}_{B}=\underline{\hat{x}}_{B}-\underline{x}
$$

$\mathrm{E}_{B}$ is the covarlance matrix associated with $\underline{e}_{B}$.

The unbiased measurement deviation vector which includes the fictitious measurements is $\underline{\mathrm{m}} \mathrm{U}$

$$
\underline{\underline{m}}_{U}=\left[\begin{array}{l}
\underline{\underline{m}}_{\mathrm{B}} \\
\underline{x}
\end{array}\right]
$$

The fictitious measurements are assumed to be the components of the state vector $\underline{x}$. The observed unbiased measurement vector is

$$
\underline{\underline{m}}_{U}=\left[\begin{array}{l}
\underline{\underline{m}}_{B} \\
\underline{0}
\end{array}\right]
$$

$\underline{x}$

The $(k+n)-b y-n$ matrix ${ }_{U}$ relates $\underline{m}_{U}$ to

$$
\underline{m}_{U}={ }^{*}{ }_{U} \underline{x}
$$

where

$$
{ }_{\mathrm{H}_{U}}^{*}=\left[\begin{array}{c}
*_{\mathrm{B}}^{*} \\
\mathrm{I}
\end{array}\right]
$$

The measurement uncertainty vector $\underline{u}_{U}$ is

$$
\begin{aligned}
\underline{u}_{U} & =\underline{\tilde{m}}_{U}-\underline{m}_{U} \\
& =\left[\begin{array}{c}
\underline{u}_{B} \\
-\underline{x}
\end{array}\right]
\end{aligned}
$$

filter $\stackrel{*}{\mathrm{~F}}_{\mathrm{U}}$ satiafy the equation

$$
\underline{\underline{x}}_{U}=\stackrel{F}{U}_{U} \underline{\tilde{m}}_{U}
$$

$\stackrel{*}{F}_{U}$ is an $n-b y-(k+n)$ matrix, whilo $\stackrel{*}{F}_{B}$ is an $n-b y-k$ matrix. If ${ }_{B}$ is given, then

$$
\stackrel{*}{\mathrm{~F}}_{\mathrm{U}}=\left[\begin{array}{ll}
\stackrel{*}{\mathrm{~F}}_{\mathrm{B}} & \stackrel{*}{\mathrm{~F}}_{\mathrm{R}}
\end{array}\right]
$$

where the residual matrix ${ }_{F_{R}}^{*}$ is an $n-b y-n$ matrix, It will now be shown that if $\mathrm{F}_{U}$ is to be unbiased, then

$$
\stackrel{*}{F}_{R}=\stackrel{*}{I}-\stackrel{*}{F}_{B} \stackrel{*}{H_{B}}
$$

For an unbiased estimate,

$$
\stackrel{*}{\mathrm{~F}}_{\mathrm{U}} \stackrel{*}{\mathrm{H}}_{\mathrm{U}}=*
$$

From Equations $(10-7)$ and $\left(10^{\circ}-11\right)$,

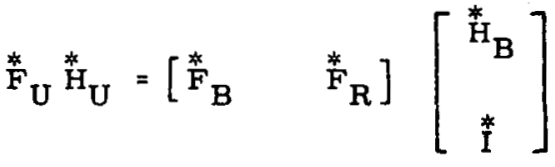

$$
\begin{aligned}
& =\stackrel{*}{F}_{B}{\stackrel{*}{H_{B}}}_{B}+\stackrel{*}{F}_{R}
\end{aligned}
$$

When $(10-13)$ and $(10-14)$ are solved for ${ }_{R^{\prime}}$, Equation $(10-12)$ is obtained.

By Equation (7-4), the unbiased covariance matrix ${ }_{U}$ is given by

$$
{\stackrel{*}{E_{U}}}=\stackrel{*}{F}_{U}{\stackrel{*}{U_{U}}}^{*}{ }^{*}{ }_{U}^{T}
$$

The uncertainty vector $\underline{e}_{B}$ can be written in terms of the unbiased filter characteristics by utilizing Equations (10-8), (10-11), and (10-12). 


$$
\begin{aligned}
\underline{e}_{B} & =\stackrel{*}{F}_{B} \stackrel{*}{H}_{B} \underline{x}+\stackrel{*}{F}_{B} \underline{u}_{B}-\underline{x} \\
& =\stackrel{*}{F}_{B} \underline{u}_{B}-\stackrel{*}{F}_{R} \underline{x} \\
& =\stackrel{*}{F}_{U}\left[\underline{u}_{B}\right]=\stackrel{*}{F}_{U} \underline{u}_{U}
\end{aligned}
$$

Finally,

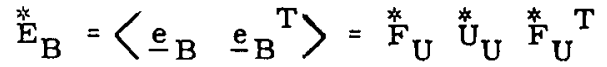

By comparing (10-15) with $(10-17)$, it is seen that

$$
\stackrel{\mathrm{x}}{\mathrm{t}}_{\mathrm{U}}=\stackrel{\mathrm{x}}{\mathrm{E}}_{\mathrm{B}}
$$

By reversing the argument presented in Equations (10-11) through (10-18), it may be shown that if $\mathrm{F}_{U}$ is given and ${ }_{\mathrm{F}}^{*} \mathrm{~F}_{\mathrm{B}}$ is obtained by deleting the last $n$ columns of $\mathrm{F}_{U}$, then the biased and unbiased covariance matrices obtained satisfy Equation $(10-18)$.

Thus, irrespective of whether one starts with a biased or unbiased filter, a filter of the other type can be constructed such that the two filters have identical matrices.

Deleting the last $n$ columns of the maximum likelihood filter ${ }_{\mathrm{ML}}$ ' obtained with the use of fictitious measurements yields the optimum biased

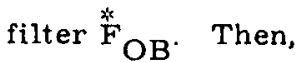

$$
\mathrm{E}_{\mathrm{OB}} \leq \mathrm{E}_{\mathrm{B}}
$$

where $\mathrm{E}_{\mathrm{B}}$ is the covariance matrix associated with the estimation uncertainty obtained by the use of any other $n$-by-k filter $F_{B}$. This is true because there is an $n$-by- $(n+k)$ unbiased filter $F_{B}{ }^{\prime}$ with covariance matrix $E_{B}$, and by the Gauss-Markov theorem

$$
\mathrm{E}_{\mathrm{B}} \geq \mathrm{E}_{\mathrm{ML}}{ }^{\prime}=\mathrm{E}_{\mathrm{OB}}
$$

Therefore, all the expressions developed in Sections 8 and 9 relating optimum unbiased filters to other unbiased filters are also applicable to the relationship between optimum biased filters and other biased filters.

\section{APPENDIX A}

A symmetric $r$-by- $r$ matrix $M$ is said to be positive definite if the quadratic form

$$
\underline{\mathrm{x}}^{\mathrm{T}} \stackrel{\mathfrak{k}_{\mathrm{M}}^{\mathrm{M}}}{\mathrm{x}}>0
$$

for every non-zero vector $\underline{x}$. If $\underline{x}^{T} \stackrel{x}{M}$ is greater than or equal to zero but not necessarily greater than zero for every non-zero vector $\underline{x}, \underline{M}$ is said to be positive semidefinite.

The principal subdeterminants of a matrix may be used to test whether or not the matrix is positive definite. The principal subdeterminant $m_{k}$ of $\mathrm{M}$ is defined as the determinant of the $\mathrm{k}-\mathrm{by}-\mathrm{k}$ matrix formed from the elements in the first $k$ rows and the first $k$ columns of $\stackrel{M}{M}$. For example, if the element in the $i-$ th row and $j$-th column of $\stackrel{k}{M}$ is designated $\mathrm{M}_{\mathrm{ij}}$;

$$
\begin{array}{r}
m_{1}=M_{11} \\
m_{2}=\left|\begin{array}{ll}
M_{11} & M_{12} \\
M_{21} & M_{22}
\end{array}\right|
\end{array}
$$

and

$$
\mathrm{m}_{\mathbf{r}}=\left|\frac{\lambda_{\mathrm{k}}}{\mathrm{M}}\right|
$$

M is positive definite if and only if all of its principal subdeterminants are positive. If some of the principal subdeterminants are zero but none are negative, $\stackrel{\star}{M}$ is positive semidefinite.

\section{APPENDIX B}

Theorem: If $\mathrm{A}$ is a positive semidefinite r-by- $r$ matrix and $B$ is an arbitrary $r-b y-s$ matrix, then the s-by-s matrix ${ }_{B} \mathrm{~T} \stackrel{*}{\mathrm{~A}}$ is positive semidefinite.

Proof: Let $\underline{z}$ be an arbitrary $s$-dimensional vector and let

$$
\underline{y}=\stackrel{\text { t }}{\mathbf{z}}
$$

Then

$$
\underline{y}^{\mathrm{T}} \stackrel{*}{\mathrm{~A}} \underline{\mathrm{y}}=\underline{\mathrm{z}}^{\mathrm{T}}{ }_{\mathrm{B}}^{\mathrm{T}} \stackrel{\mathrm{A}}{\mathrm{A}} \underline{\mathrm{z}} \geq 0
$$

because A $A$ is positive semidefinite. Hence $\mathrm{B}^{\mathrm{T}} \mathrm{A}$ is positive semidefinite. 


\section{APPENDIX C}

Theorem: Let $\stackrel{*}{A}$ and $B$ be symmetric $r$-by$r$ matrices such that

$$
\text { A } \geq \stackrel{B}{B}>0
$$

Then,

$$
|A| \geq \mid \text { B } \mid>0
$$

Also,

$$
|=| \text { 劳 } \mid
$$

only if

$$
\text { B }=\stackrel{*}{\mathbf{A}}
$$

Proof: By a matrix theorem used in the theory of vibrations (Page 47 of Reference (8)), A and $\mathrm{B}$ can be simultaneously diagonalized by a nonsingular matrix $\mathrm{M}$.

$$
\begin{aligned}
& \mathrm{M}^{\mathrm{T}} \stackrel{*}{\mathrm{~A}} \stackrel{*}{\mathrm{M}}=\stackrel{k}{\mathrm{D}} \\
& \text { M }^{\mathrm{T}} \mathrm{B}_{\mathrm{M}}^{*}=\mathrm{N}_{\mathrm{I}}^{*}
\end{aligned}
$$

where $D$ is a diagonal matrix whose i-th diagonal element is $d_{i}$, W L the-inverse of ${ }^{*}$.

$$
\text { A }-{ }_{B}^{*}=*^{T} \text { (D }
$$

Let $\underline{h}_{k}$ be a column vector whose $k$-th component is one and whose other components are all zeros. Let

$$
\mathbf{p}_{\mathrm{k}}=\stackrel{*}{M} \underline{\mathrm{h}}_{\mathrm{k}}
$$

Then

$$
\mathrm{p}_{\mathrm{k}}^{\mathrm{T}}(\stackrel{*}{\mathrm{~A}}-\stackrel{*}{\mathrm{~B}}) \mathrm{p}_{\mathrm{k}}=\left(\mathrm{d}_{\mathrm{k}}-1\right) \geq 0
$$

The inequality holds because $\left(A^{*}-B^{*}\right)$ is positive semidefinite. From (C-9),

$$
d_{k} \geq 1
$$

for $k^{\prime}=1, \ldots \ldots r$.

The determinants of $\mathrm{B}$ and $A$ are given by

$$
\begin{gathered}
|\stackrel{*}{B}|=\left|N_{N}^{*}\right|^{2} \\
|A|=d_{1} d_{2} \ldots d_{r}|N|^{2}=d_{1} d_{2} \ldots d_{r}|\stackrel{B}{B}|
\end{gathered}
$$

(C-12)

In consequence of $(C-10),(C-11)$, and $(C-12)$,

$$
\begin{aligned}
& |\mathrm{A}| \geq|\mathrm{B}|>0 \quad(\mathrm{C}-13) \\
& \text { If }|B|=|A|, d_{1}^{*} \cdot d_{2}=\ldots=d_{r}=1 \text {, }
\end{aligned}
$$
and from (C-7)

$$
\stackrel{*}{\mathrm{~A}}-\stackrel{*}{\mathrm{~B}}=\stackrel{*}{0}
$$

\section{APPENDIX D}

Theorem: Let and be positive semidefinite symmetric $r$-by-r matrices, then

$$
\operatorname{tr}\left(\stackrel{A}{*}^{*}\right) \geq 0
$$

If $A$ is positive semidefinite and $\frac{1}{B}$ is positive definite, then

$$
\operatorname{tr}(\stackrel{\star}{A})=0
$$

only if

$$
\stackrel{*}{A}=*
$$

Proof: Since A positive semidefinite, it can be diagonalized by an orthogonal matrix $\mathrm{M}$.

$$
\vec{D}=\stackrel{*}{M}^{T} \stackrel{*}{A}
$$

where $D$ is a diagonal matrix whose $i-t h$ diagonal element is $d_{i}$. The fact that $A$ is positive semidefinite implies that

$$
d_{i} \geq 0
$$

for $i=1, \ldots . r$. Let $D_{1}$ be a diagonal matrix whose $i$-th diagonal element is $d_{i} 1 / 2$ Then

$$
\text { D }=D_{1}^{*} D_{1}^{T}
$$

Now, let

$$
\stackrel{*}{\mathrm{~A}_{1}}=\stackrel{*}{\mathrm{M}} \stackrel{*}{\mathrm{D}_{1}} \stackrel{*}{\mathrm{M}} \mathrm{T}
$$

Because ${ }^{*}$ is orthogonal, it follows that

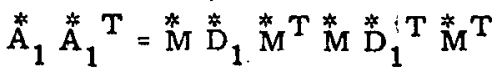

$$
\begin{aligned}
& =\stackrel{*}{\mathrm{M}} \stackrel{\mathrm{s}}{\mathrm{D}} \mathrm{M}^{\mathrm{T}}=\stackrel{*}{\mathrm{~A}}
\end{aligned}
$$

Since $\operatorname{tr}(\stackrel{*}{\mathrm{~B}})=\operatorname{tr}(\mathrm{B} \stackrel{*}{\mathrm{~A}})$,

$$
\begin{aligned}
\operatorname{tr}(\mathrm{A}) & =\operatorname{tr}\left[\mathrm{A}_{1}^{*}\left(\mathrm{~A}_{1}^{*} \mathrm{~T}\right)\right] \\
& =\operatorname{tr}\left(\mathrm{A}_{1}^{*} \mathrm{~T}{ }_{\mathrm{B}}^{*}\right)
\end{aligned}
$$

By Appendix $B$, the matrix $\stackrel{*}{A}_{1}^{T}{ }_{B}^{*} \stackrel{A}{A}_{1}$ is positive semidefinite and therefore has non-negative diagonal elements. Thus, in view of $(D-9)$,

$$
\operatorname{tr}(\stackrel{*}{\mathrm{~B}}) \geq 0
$$

To prove the second part of the theorem, let $A_{1}$ be partitioned into column vectors $\underline{s}_{1}, \ldots \underline{\mathrm{B}}$

$$
\stackrel{*}{A}_{1}=\left[\underline{B}_{1} \ldots \underline{B}_{\mathbf{r}}\right]
$$

(D-11) 
Then if $\operatorname{tr}(\stackrel{*}{\mathrm{~A}} \mathrm{~B})=0$, it follows that

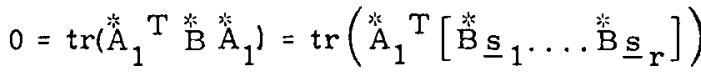

$$
\begin{aligned}
& =\sum_{i=1}^{r} \underline{s}_{i}{ }^{T} \stackrel{*}{B} \underline{s}_{i}
\end{aligned}
$$

Since $\stackrel{\text { 等 }}{\mathrm{B}}$ is positive definite,

$$
\underline{s}_{i}{ }^{T} \underline{s}_{i} \geq 0
$$

and hence

$$
\underline{s}_{i}{ }^{T} \underline{s}_{i}=0
$$

by Equation (D-21). Again using the fact that $B$ is positive definite, it follows that

$$
\underline{\mathrm{s}}_{\mathrm{i}}=\underline{0}
$$

for $i=1, \ldots, r_{1}$ thus

$$
\mathrm{A}_{1}=\mathrm{*}
$$

and by $(\mathrm{D}-8)$

$$
\ddot{A}=\stackrel{*}{A}_{1} \stackrel{*}{A} T=\stackrel{*}{O}
$$

\section{$\underline{\text { References }}$}

1. McLean, J.D., Schmidt, S.F., and McGee, L. A., "Optimal Filtering and Linear Prediction Applied to a Midcourse Navigation System for the Circumlunar Mission," NASA Tech. Note D-1208, 1962.

2. Carr, R.E. and Hudson, R.H., "Tracking and Orbit Determination Program of the Jet Propulsion Laboratory," JPL Technical Report No. 32-7, Feb. 1960.

3. Smith, G. L., Schmidt, S.F., and McGee, L. A., "Application of Statistical Filter Theory to the Optimal Estimation of Position and Velocity on board a Circumlunar Vehicle," NASA Tech. Rept. R-135, 1962.

4. Battin, R.H., "A Statistical Optimizing Navigation Procedure for Space Flight," ARS Journal, Vol. 32 No. 11, Nov. 1962, pp. 1681 - 1696.

5. Stern, R. G., "Interplanetary Midcourse Guidance Analysis," Sc. D. thesis in Department of Aeronautics and Astronautics, M. I. T., May 1963.
6. Noton, A.R.M., "Interplanetary Post-Injection Guidance," JPL External Publication No. 653, June 1959.

7. Bellman, R., "Introduction to Matrix Analysis," McGraw-Hill Book Co., New York, 1960.

3. Hildebrand, F.B., Methods of Applied Mathematics, Prentice-Hall, Inc., New York, 1952. 